\title{
Sífilis congénita en México. Análisis de las normas nacionales e internacionales desde la perspectiva del diagnóstico de laboratorio
}

\author{
Antonia Herrera-Ortiz, Hugo López-Gatell, Santa García-Cisneros, María Alejandra Cortés-Ortiz, \\ María Olamendi-Portugal, Jennifer Hegewisch-Taylor y Miguel Ángel Sánchez-Alemán* \\ Instituto Nacional de Salud Pública, Centro de Investigación sobre Enfermedades Infecciosas, Cuernavaca, Morelos, México
}

\section{Resumen}

Introducción: La sífilis congénita continúa siendo un problema de salud pública en México. Objetivo: Evaluar las similitudes y diferencias entre normas nacionales, guías y documentos internacionales relacionados con la detección de sífilis en embarazadas y sífilis congénita. Método: Se elaboraron dos algoritmos basados en las normas sobre atención de la mujer durante el embarazo y sobre prevención y control de infecciones de transmisión sexual. A partir de la guía de Centers for Disease Control and Prevention (CDC) se realizaron algoritmos sobre sífilis durante el embarazo, sífilis en recién nacido y contactos sexuales. Resultados: La norma sobre embarazo menciona que la prueba de sífilis debe efectuarse a toda mujer embarazada en su primer contacto o durante el parto, sin especificar pruebas diagnósticas. La norma oficial mexicana (NOM) sobre infecciones de transmisión sexual menciona el algoritmo tradicional para detección de sífilis, seguimiento al tratamiento, coinfección con virus de inmunodeficiencia humana y criterios de sífilis congénita. CDC recomienda algoritmo reverso, título de anticuerpos, tratamiento y seguimiento como parte del diagnóstico. Conclusiones: La eliminación de la transmisión maternoinfantil de sífilis requiere actualizar y homogeneizar las NOM, así como el estudio de mortinatos y de recién nacidos de madres con sífilis.

PALABRAS CLAVE: Sífilis congénita. Serodiagnóstico de la sífilis. Complicaciones infecciosas del embarazo. Guías de práctica clínica.

\begin{abstract}
Introduction: Congenital syphilis continues to be a public health problem in Mexico. Objective: To assess the similarities and differences between national standards, guidelines and international documents related to the detection of syphilis in pregnant women and congenital syphilis. Method: Two algorithms were developed based on the standard of female care during pregnancy and on the standard for prevention and control of sexually transmitted infections. Based on the Centers for Disease Control ( $C D C$ ) guidelines, algorithms were developed for syphilis during pregnancy, syphilis in the newborn and sexual contacts. Results: The standard for pregnancy mentions that syphilis testing should be carried out in every pregnant woman on her first contact or at delivery, without diagnostic tests being specified. The Official Mexican Standard (NOM) on sexually transmitted infections mentions the traditional algorithm for syphilis detection, treatment follow-up, coinfection with human immunodeficiency virus and congenital syphilis criteria. The CDC recommend reverse algorithm, antibody titer, treatment and follow-up as part of diagnosis. Conclusions: The elimination of mother-to-child transmission of syphilis requires NOMs updating and homogenizing, as well as the study of stillbirths and neonates born to mothers with syphilis.
\end{abstract}

KEY WORDS: Congenital syphilis. Serodiagnosis of syphilis. Infectious complications of pregnancy. Clinical practice guidelines.

Correspondencia:

*Miguel Ángel Sánchez-Alemán

E-mail: msanchez@insp.mx
Fecha de recepción: 23-10-2018

Fecha de aceptación: 19-03-2019

DOI: 10.24875/GMM.19004779
Gac Med Mex. 2019;155:464-472

Disponible en PubMed

www.gacetamedicademexico.com 


\section{Introducción}

Treponema pallidum es una bacteria que puede atravesar la placenta, por lo que una mujer embarazada con sífilis puede presentar aborto espontáneo, muerte fetal, parto prematuro y su producto, sífilis congénita (SC). La SC es una forma severa del padecimiento, que puede ocurrir en cualquier estadio clínico de la enfermedad materna y también depende de la semana de gestación. ${ }^{1,2}$

En América Latina y el Caribe, la sífilis materna y la SC constituyen un grave problema de salud pública: la prevalencia de sífilis en mujeres embarazadas oscila entre 0.1 y $7.0 \%$; se estimaron 1.7 casos de SC por 1000 nacidos vivos en 2015. ${ }^{3}$ Para México, el número de casos reportados de SC aumentó de 43 casos en 2013 a 121 en 2017. ${ }^{4}$ Una encuesta nacional en México encontró $6.6 \%$ de anticuerpos contra Treponema pallidum en mujeres con antecedente de hijos nacidos muertos y $2.6 \%$ de anticuerpos en mujeres sin hijos nacidos muertos, lo que sugiere una asociación entre Treponema pallidum y abortos. ${ }^{5}$ Diversos estudios en mujeres embarazadas en México han detectado prevalencias de sífilis entre 0.26 y $2.3 \% .^{6-9}$

En 1994, la Organización Panamericana de la Salud realizó el primer llamado para la eliminación de la SC en las Américas; en 2009, esa organización y el Fondo de las Naciones Unidas para la Infancia lanzaron una iniciativa para la eliminación de la transmisión maternoinfantil (ETMI), del virus de inmunodeficiencia humana (VIH) y de la SC en América Latina y el Caribe; en 2017 se lanzó ETMI-plus, que adiciona la eliminación maternoinfantil de la hepatitis $B$ y la enfermedad de Chagas. ${ }^{10-11} \mathrm{La}$ iniciativa buscó reducir la incidencia de SC a 0.5 casos o menos (incluyendo mortinatos) por 1000 nacidos vivos para 2015; esta meta ha sido cumplida en 20 naciones, de los cuales Cuba fue la primera en recibir la validación de la Organización Mundial de la Salud. México es uno de los países que reportan progreso hacia la eliminación, si bien no ha alcanzado la meta. ${ }^{12}$

La Organización Panamericana de la Salud considera implementar pruebas de diagnóstico rápido, ofrecer tratamiento inmediato e involucrar a las parejas de las pacientes con sífilis, como medidas cruciales para poner fin a la transmisión maternoinfantil para el 2030.12 Existen recomendaciones en documentos internacionales y en las diversas normas oficiales mexicanas que orientan sobre los procedimientos relacionados con la prevención, detección y tratamiento de la sífilis en mujeres embarazadas. El objetivo de este estudio es presentar un análisis de la normatividad nacional e internacional, enfocado en el diagnóstico de laboratorio de sífilis en mujeres embarazadas como primer paso para la prevención de la transmisión vertical de sífilis en México.

\section{Método}

En el proyecto Análisis longitudinal de los determinantes y diseño de una estrategia integral de prevención de la transmisión vertical de VIH y sífilis congénita en México se revisaron documentos internacionales y nacionales para identificar las intervenciones involucradas en el diagnóstico de sífilis materna como medida de prevención de la SC. Un equipo de trabajo, de al menos tres personas para cada documento, leyó cada una de las normas oficiales mexicanas (NOM). Se comenzó con dos normas, la NOM-007SSA2-2016, para la atención de la mujer durante el embarazo, parto y puerperio y del recién nacido ${ }^{13} \mathrm{y}$ la NOM-039-SSA2-2014, para la prevención y control de las infecciones de transmisión sexual. ${ }^{14}$ En estas se refieren normas adicionales, que fueron revisadas y de las cuales se seleccionaron seis, para su análisis a fondo. El criterio de selección fue que contuvieran uno de los siguientes términos: sífilis, treponema, infección de transmisión sexual (ITS), enfermedad de transmisión sexual (ETS) ० $\mathrm{VIH} .{ }^{15-20}$ A partir de la revisión de las dos primeras normas se realizaron dos esquemas, en los que se presentan las actividades que deben realizarse.

Posteriormente se revisó el catálogo maestro de guías de prácticas clínicas (GPC) elaboradas por la Secretaría de Salud, en el que se encontraron y revisaron dos GPC: Enfermedades de transmisión sexual en el adolescente y adulto que producen úlceras genitales: herpes, sifilis, chancroide, linfogranuloma venéreo y granuloma inguinal; ${ }^{21}$ y Diagnóstico, tratamiento y seguimiento de la sífilis congénita en el primero y segundo nivel de atención. ${ }^{22}$ También se buscaron documentos de la Organización Mundial de la Salud y de la Organización Panamericana de la Salud; solo se identificaron documentos estratégicos, sin elementos operativos para la detección de sífilis mediante pruebas de laboratorio. La búsqueda se amplió a Centers for Disease Control an Prevention (CDC) de Estados Unidos; encontramos un documento que establece los procedimientos relativos 
Tabla 1. Listado de normas oficiales mexicanas asociadas con atención del embarazo y sífilis

\begin{tabular}{|c|c|}
\hline Norma & Objetivo de la norma \\
\hline $\begin{array}{l}\text { NOM-007-SSA2-2016, para } \\
\text { la atención de la mujer } \\
\text { durante el embarazo, parto y } \\
\text { puerperio y del recién nacido }\end{array}$ & $\begin{array}{l}\text { Establecer criterios mínimos para la atención } \\
\text { médica a la mujer durante el embarazo, parto y } \\
\text { puerperio normal y a la persona recién nacida. }\end{array}$ \\
\hline $\begin{array}{l}\text { NOM-010-SSA2-2018, para } \\
\text { la prevención y el control de } \\
\text { la infección por virus de la } \\
\text { inmunodeficiencia humana }\end{array}$ & $\begin{array}{l}\text { Establecer y actualizar los métodos, principios } \\
\text { y criterios de operación de las actividades } \\
\text { relacionadas con la prevención y control, que } \\
\text { abarcan la detección, el diagnóstico oportuno, } \\
\text { la atención y tratamiento médico de la infección } \\
\text { por VIH. }\end{array}$ \\
\hline
\end{tabular}

NOM-017-SSA2-2012, para la vigilancia epidemiológica

NOM-034-SSA2-2013. Para la prevención y control de los defectos al nacimiento

NOM-039-SSA2-2014, para la prevención y control de las infecciones de transmisión sexual

NOM-046-SSA2-2005, violencia familiar, sexual y contra las mujeres. Criterios para la prevención y atención

NOM-253-SSA1-2012, para la disposición de sangre humana y sus componentes con fines terapéuticos
Establecer lineamientos y procedimientos de operación, así como los criterios para la aplicación de la vigilancia epidemiológica en padecimientos, eventos y situaciones de emergencia que afectan o ponen en riesgo la salud humana.

Establecer criterios y especificaciones para la prevención, diagnóstico, tratamiento y control de los defectos al nacimiento. Se incluyen los principales defectos prevenibles o susceptibles de diagnóstico temprano, así como medidas de prevención y control que puedan tener impacto epidemiológico en las tasas de morbilidad y mortalidad perinatal.

Establecer y uniformar los procedimientos y criterios de operación para la prevención y el control de las infecciones de transmisión sexual.

Establecer criterios en la detección, prevención, atención médica y orientación que se proporciona a las y los usuarios de servicios de salud en general, y en particular a quienes se encuentren involucrados en situaciones de violencia familiar o sexual, así como en la notificación de casos.

Establecer actividades, criterios, estrategias y técnicas operativas en relación con la disposición de sangre humana y sus componentes con fines terapéuticos

\section{Contenido respecto a sífilis}

Se busca impedir la transmisión perinatal de sífilis y $\mathrm{VIH}$, mediante su detección y tratamiento oportunos. Establece que se debe realizar prueba de laboratorio para detectar sífilis en la embarazada y prevenir SC (5.2.1.14.7; 5.2.1.14.8).

Establece que a la mujer en edad reproductiva o embarazada y a su pareja se les debe ofertar detección de VIH y sífilis (5.13-5.15).

La detección de VIH y sífilis en mujeres embarazadas debe realizarse en al menos dos momentos durante el embarazo: una prueba inicial en las primeras 12 semanas de gestación o al momento de captarla y una segunda prueba antes del parto (6.3.4.2).

Establece la SC como una infección de notificación inmediata (7.12), a la sífilis adquirida y congénita como objeto de notificación semanal (7.14.5.4). Son objeto de estudio epidemiológico de caso, los casos o defunciones por SC (8.1). La SC requiere confirmación de laboratorio (12.6.3.3)

Se define la SC como la infección ocasionada por Treponema pallidum transmitida de la madre al producto por circulación transplacentaria. Se establece el uso de VDRL o pruebas rápidas para el diagnóstico prenatal y tamiz neonatal de sífilis, y uso de FTA-ABS en el seguimiento posnatal (8.2.1. Cuadro 4)

Establece los lineamientos para la detección (métodos diagnósticos), tratamiento y seguimiento de la sífilis en el embarazo (7.4.5). Así como los criterios para el diagnóstico de SC en recién nacidos (7.4.5.6).

En el caso de maltrato en mujeres embarazadas, valorar entre otras, infecciones genitales recurrentes durante la gestación, con el apoyo de exámenes de laboratorio y gabinete y estudios especiales (6.2.1.6.)

La sífilis y otras enfermedades transmisibles por transfusión constituyen motivo de diferimiento para donar sangre o componentes sanguíneos hasta por 12 meses (6.10.6.3). Lineamientos para el diagnóstico de Treponema pallidum, así como las pruebas que pueden emplearse en tamizaje (VDRL/RPR/IC/EIA) y confirmación (HA/FTA-ABS/IFI/INM) (9.4.9)

SC = sífilis congénita, $\mathrm{VIH}=$ virus de inmunodeficiencia humana, ITS = infecciones de transmisión sexual, IC = inmunocromatografía, EIA = ensayo inmunoenzimático, $\mathrm{HA}=$ hemaglutinación, FTA-ABS = absorción de anticuerpos fluorescentes, IFI = inmunofluorescencia, INM = inmovilización de treponemas.

a la prevención, diagnóstico y tratamiento de sífilis en mujeres embarazadas y SC, el cual se utilizó como una referencia internacional. Se realizaron tres esquemas de actividades, correspondientes al diagnóstico de sífilis durante el embarazo, diagnóstico de sífilis de recién nacidos de madre seropositiva y diagnóstico a contactos sexuales de personas con diagnóstico de sífilis. ${ }^{23}$

\section{Resultados}

Se analizaron ocho normas oficiales mexicanas; en la Tabla 1 se describen los aspectos clave relacionados con la sífilis. El algoritmo de la NOM-007-SSA2-2016 de atención durante el embarazo (Figura 1) pone de manifiesto que debe realizarse la prueba de sífilis a toda embarazada en su primer contacto con el sector salud o durante el parto, si este fue su primer contacto. 


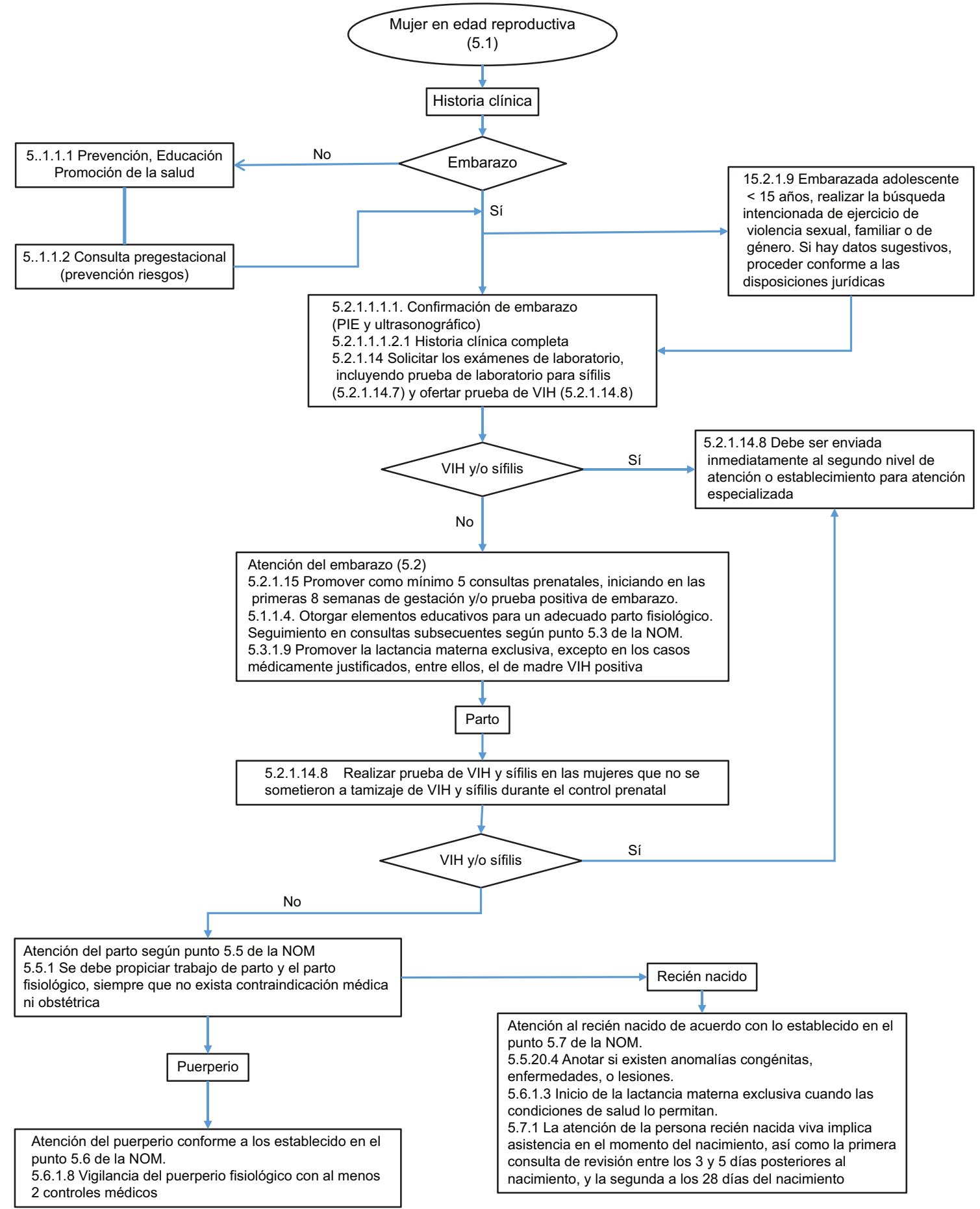

Figura 1. Algoritmo con base en la Norma oficial mexicana NOM-007-SSA2-2016, para la atención de la mujer durante el embarazo, parto y puerperio y de la persona recién nacida.

Esta norma no especifica el tipo de pruebas para la detección de sífilis, tampoco el tratamiento y seguimiento del recién nacido o de contactos. En la NOM039-SSA2-2014 sobre ITS (Figura 2) se refiere que el diagnóstico de sífilis depende de su etapa clínica, se señala el tipo de pruebas por realizar, la periodicidad para darle seguimiento al tratamiento y la coinfección con $\mathrm{VIH}$; se establecen las acciones para mujeres embarazadas con sífilis y se menciona la necesidad de realizar pruebas presuntivas y confirmatorias en cada trimestre, incluso después del parto. El tratamiento se establece conforme a la etapa clínica y se enlistan los 


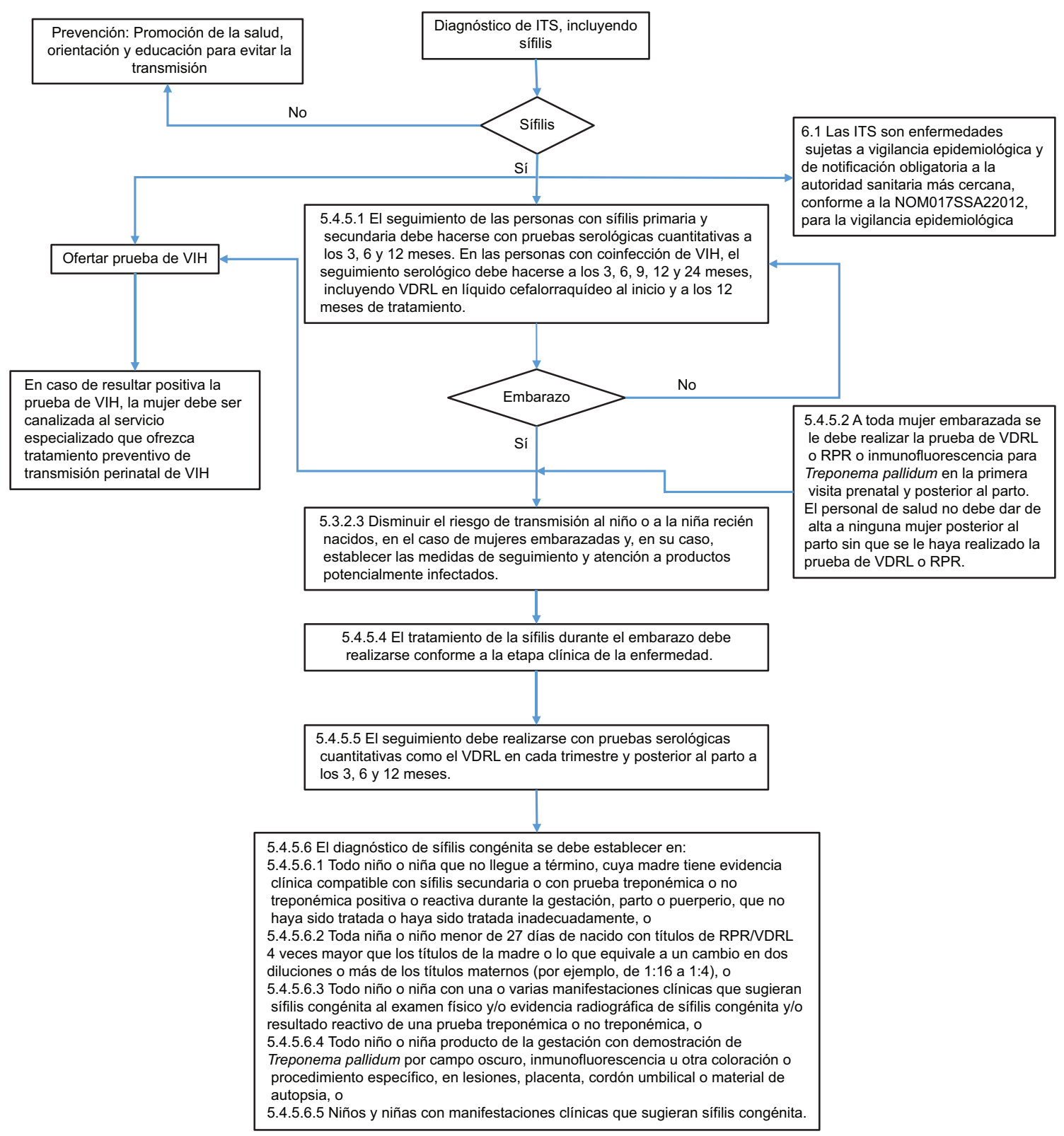

5.4.5.7 El seguimiento de la niña o niño de madre con sífilis debe efectuarse al mes, 2, 4, 6 y 12 meses.

Figura 2. Algoritmo con base en la Norma oficial mexicana NOM-039-SSA2-2014, para la prevención y control de las infecciones de transmisión sexual.

criterios para descartar SC, así como el seguimiento para los casos presuntivos o confirmados.

La GPC Enfermedades de transmisión sexual en el adolescente $y$ adulto que producen úlceras genitales: herpes, sifilis, chancroide, linfogranuloma venéreo y granuloma inguinal carece de un apartado específico para mujeres embarazadas; menciona evidencias y recomendaciones para el diagnóstico, manejo y tratamiento de las ITS ulcerativas en adolescentes y adultos. Indica que debe realizarse tamizaje a toda mujer embarazada y que la prueba de VDRL con título 1:8 es orientativa de positividad a sífilis; hace referencia a pruebas de laboratorio para detección de sífilis en personas sintomáticas. La GPC Diagnóstico, tratamiento y seguimiento de la sífilis congénita en el primero y segundo nivel de atención define caso probable, confirmado y anulado de sífilis congénita, además de óbito por sífilis. Especifica las acciones para el diagnóstico, manejo y tratamiento de SC; los lineamientos respecto al recién nacido son detallados, sin 


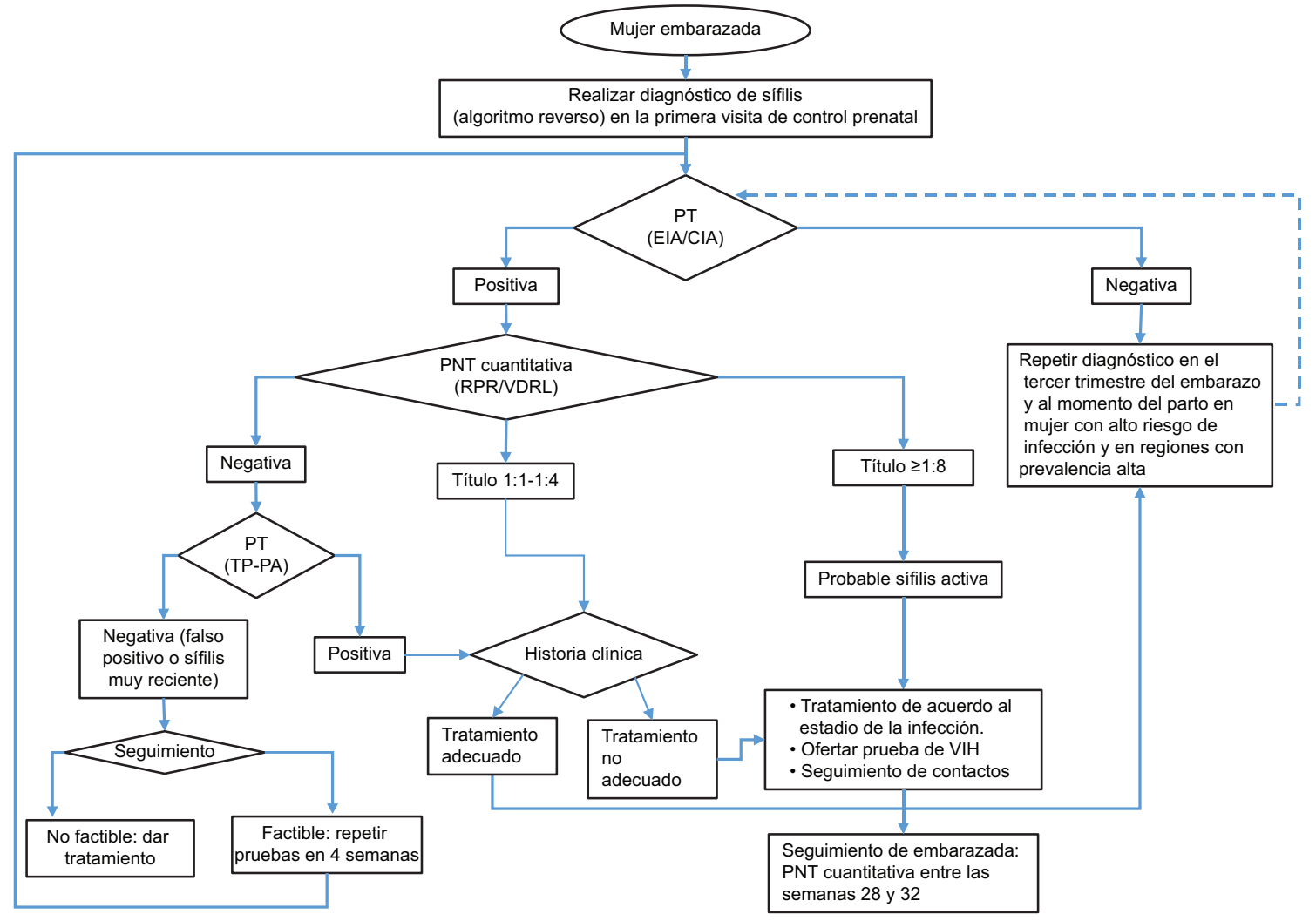

Figura 3. Algoritmo relativo a la mujer embarazada a partir de Sexually Transmitted Diseases

Treatment Guidelines, 2015, Centers for Disease Control and Prevention. PT = prueba treponémica, EIA = ensayo inmunoenzimático, $C I A=$ ensayo quimioluminiscente, $P N T=$ prueba no treponémica, $T P-P A=$ aglutinación de partículas.

embargo, se limitan a casos de SC y no a la mujer embarazada, por lo que su aplicación no tiene efecto en la prevención de la transmisión vertical de sífilis.

En Sexually Transmitted Diseases Treatment Guidelines 2015, del CDC, se establecen lineamientos de detección, tratamiento y seguimiento de ITS; en el apartado de sífilis durante el embarazo se destacan los lineamientos para el diagnóstico durante el embarazo, las premisas para el seguimiento del recién nacido y las directrices para el seguimiento de contactos. Se recomienda el uso del algoritmo reverso (Figura 3), que inicia con un ensayo treponémico, seguido de una prueba no treponémica y, de ser necesario, un ensayo treponémico confirmatorio. Adicionalmente, se consideran el título de anticuerpos, los tratamientos previos y la factibilidad del seguimiento a la embarazada. Para la prevención de SC debe realizarse el diagnóstico en la primera consulta prenatal, en la semana 28 y al momento del parto; ninguna madre ni su hijo recién nacido deben ser dados de alta sin un estatus serológico bien documentado. Esta guía considera que a cualquier mujer que presente muerte fetal después de 20 semanas debe realizarse la prueba para diagnóstico de sífilis.
Todo recién nacido de una madre seropositiva para sífilis debe mantenerse en seguimiento. El diagnóstico del neonato se basa en cuatro escenarios distintos, dependiendo de los antecedentes clínicos de la madre y del análisis físico del neonato, tal como se presenta en la Figura 4. La decisión sobre el tratamiento de SC considera:

- Identificación de sífilis en la madre.

- Tratamiento adecuado de la madre.

- Evidencia clínica, radiológica o de laboratorio de sífilis en el neonato.

- Comparación de títulos de anticuerpos, maternos versus neonato.

Es importante resaltar que esta guía no recomienda el uso de pruebas serológicas treponémicas para el recién nacido y se indica que no hay pruebas de detección de IgM confiables. Finalmente, en relación con el seguimiento de contactos, se establece que debe notificarse confidencialmente a los contactos sexuales de las personas bajo tratamiento. El seguimiento difiere en función del estadio de la enfermedad y del periodo entre el contacto sexual y el diagnóstico de sífilis. El algoritmo considera la factibilidad del seguimiento y el uso de pruebas serológicas (Figura 5). 


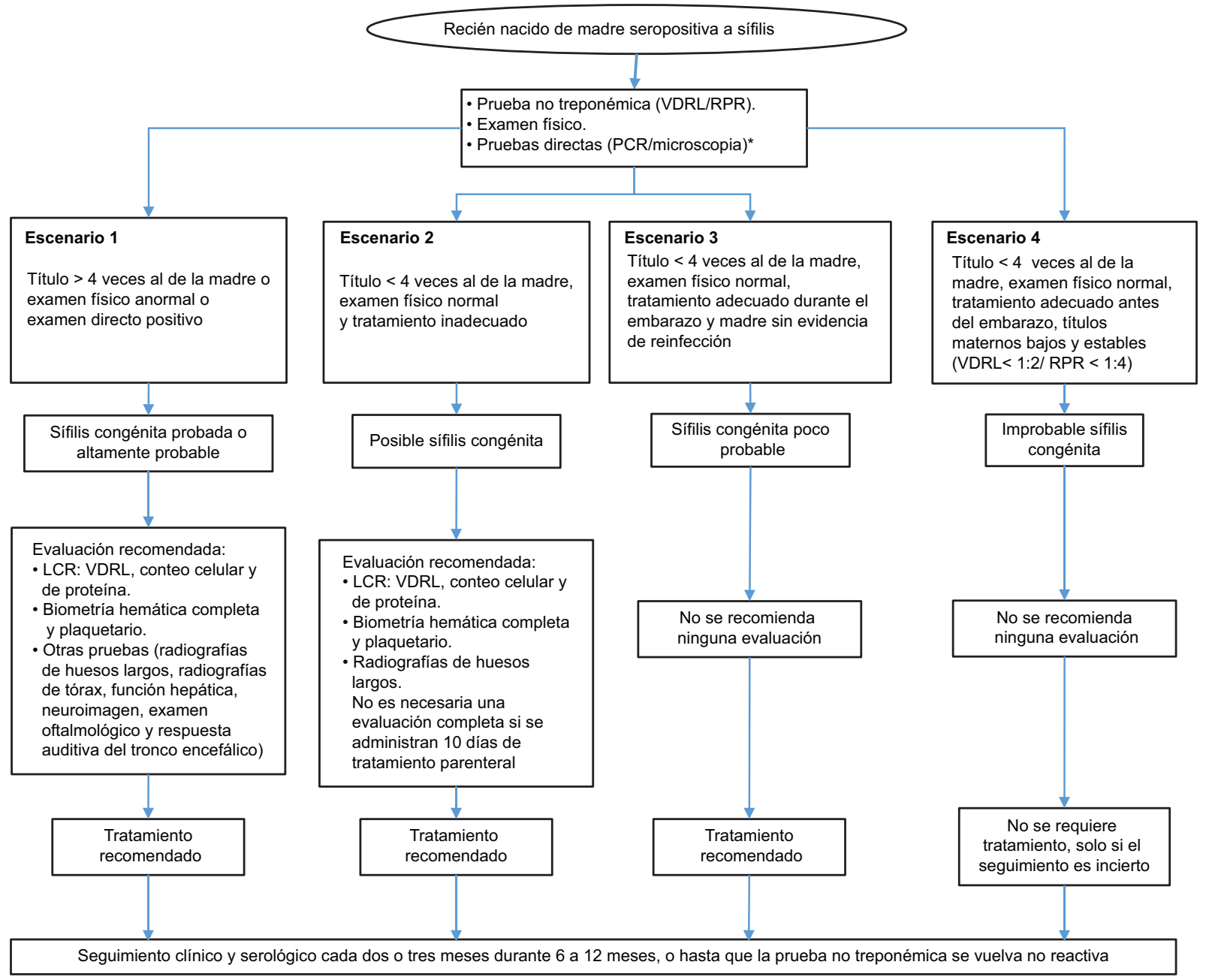

Figura 4. Algoritmo relativo al recién nacido. Adaptado de Sexually Transmitted Diseases Treatment Guidelines, 2015, Centers for Disease Control and Prevention.

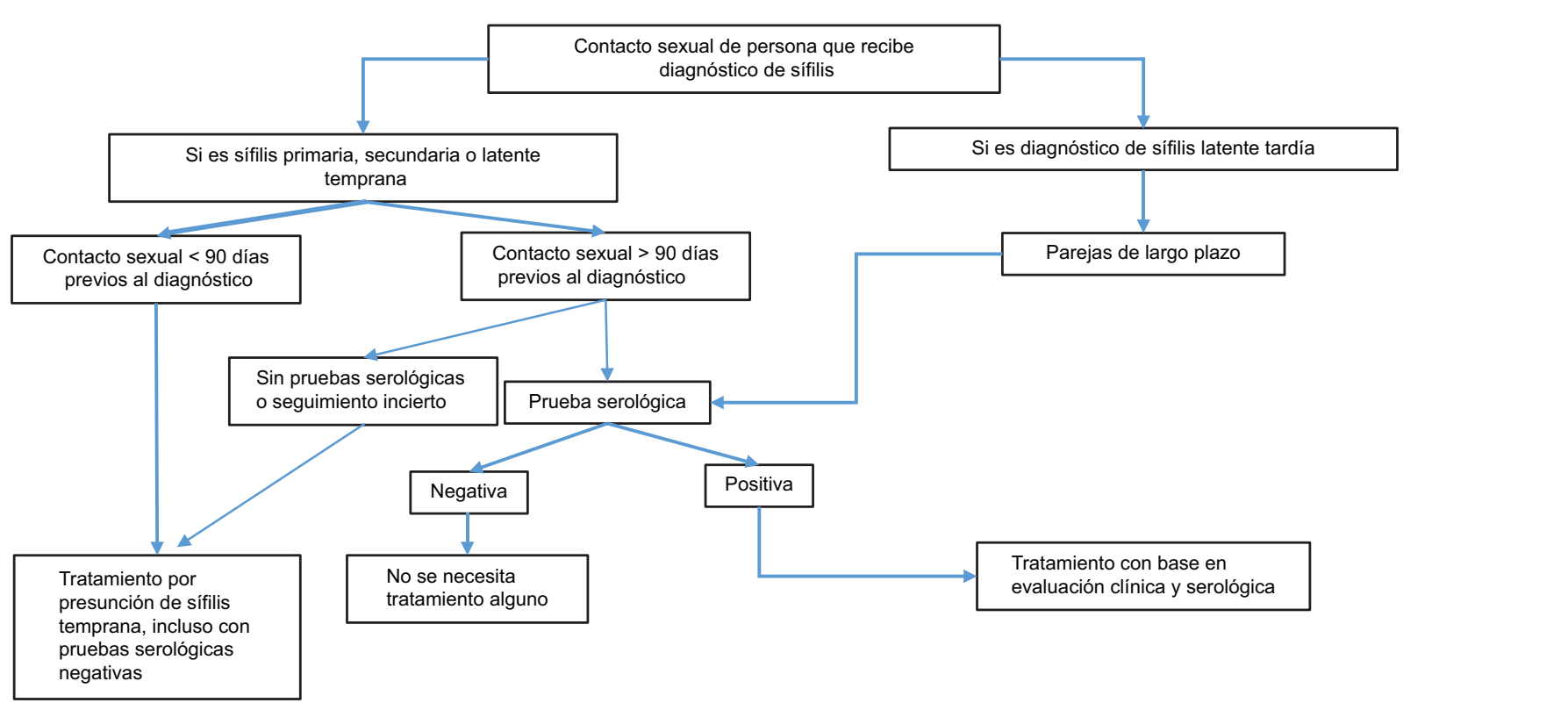

Figura 5. Seguimiento de contactos sexuales. Adaptado de Sexually Transmitted Diseases Treatment Guidelines, 2015, Centers for Disease Control and Prevention. 


\section{Discusión}

Para lograr la eliminación de la SC es esencial contar con un marco normativo actualizado, enfocado al binomio madre-hijo, similar a lo que México tiene para VIH, como se aprecia en la GPC Prevención, diagnóstico y tratamiento en el binomio madre-hijo con infección por el VIH. ${ }^{24}$ De acuerdo con lo revisado, las normas oficiales mexicanas y las GPC no parecen estar dirigidas a la prevención de la transmisión maternoinfantil de sífilis, sino a una serie de lineamientos para garantizar estándares mínimos de calidad en el país. Se carece de documentos enfocados exclusiva y específicamente a la prevención de la SC o en conjunto con $\mathrm{VIH} /$ Chagas/ hepatitis $\mathrm{B}$, que deberían considerar integralmente a la madre, al recién nacido, a los contactos sexuales y hacer énfasis en el seguimiento de los casos, para evitar la transmisión vertical y sexual.

A nivel estatal destaca el documento Guía diagnóstico y manejo de la sífilis en el embarazo y prevención de la sífilis congénita, del gobierno de Sonora, ${ }^{25}$ el cual considera el enfoque integral del binomio madre-hijo, sin embargo, está basado en la versión de 2006 de la guía del CDC sobre ITS y requiere el sustento de normas oficiales mexicanas actualizadas.

El diagnóstico oportuno de sífilis en una mujer embarazada es el primer paso para la prevención de la SC, sin embargo, las recomendaciones internacionales sugieren momentos adicionales para detectar sífilis, no solo durante la primera visita sino también durante el tercer trimestre y al momento del parto. ${ }^{23}$ En relación con el tipo de pruebas, la NOM-039-SSA2-2014 sobre ITS menciona que las de laboratorio deben realizarse conforme la etapa de la enfermedad, mediante microscopia de campo obscuro o inmunofluorescencia en sífilis primaria y mediante VDRL, RPR y FTA-ABS en la sífilis secundaria. El diagnóstico con base en el cuadro clínico soslaya los casos asintomáticos, que constituyen la mayoría, y que también pueden originar SC. ${ }^{2}$ Estas directrices en el diagnóstico de sífilis difieren de otras normas oficiales mexicanas; existen variaciones respecto al momento para el diagnóstico y las técnicas sugeridas para hacerlo. En la NOM-034-SSA2-2013 sobre defectos al nacimiento se menciona al VDRL como tamizaje prenatal y pruebas rápidas y para el seguimiento posnatal, el FTA-ABS. Finalmente, en la NOM253-SSA1-2012 sobre disposición de sangre humana, específicamente en el apartado sobre transfusión sanguínea, se mencionan diversas pruebas de tamizaje (VDRL, RPR, ensayos inmunoenzimáticos e inmunocromatográficos) y confirmatorias (hemaglutinación, absorción de anticuerpos fluorescentes, inmunofluorescencia indirecta e inmovilización del treponema).

En relación con los métodos diagnósticos, algunas pruebas directas mencionadas en las normas oficiales mexicanas se encuentran en desuso. En 2014 se realizó un estudio en 69 laboratorios y solo en dos de ellos se efectuaban pruebas de microscopia de campo oscuro. ${ }^{26}$ En la NOM-253-SSA1-2012 sobre la disposición de sangre humana resalta la mención del método de inmovilización de treponemas, que la Organización Mundial de la Salud recomendó en 1956 y que no se utiliza actualmente. ${ }^{27}$ En contraparte, existen otras pruebas directas que no se mencionan en las normas oficiales mexicanas, principalmente las moleculares, que cada vez tienen mayor difusión. ${ }^{28}$ Respecto a las pruebas confirmatorias, la NOM-253-SSA1-2012 solo menciona la FTA-ABS, tradicionalmente estándar de oro, sin embargo, en la actualidad existen otros tipos de pruebas confirmatorias como MHA-TP (ensayos de microhemaglutinación), TPPA (ensayo de aglutinación pasiva de partículas) y TPHA (ensayo de hemaglutinación), con mayor aceptación por costo, tiempo, entrenamiento y sin el requerimiento de equipos sofisticados. ${ }^{29}$

Según los resultados del programa de control de calidad para serología de sífilis, 110 laboratorios reportaron pruebas de TPPA/TPHA y solo 52 de FTA-ABS. ${ }^{30}$ Por otra parte, es necesario considerar el algoritmo reverso, iniciar con una prueba treponémica, posteriormente la titulación con pruebas no treponémicas $y$, de ser necesario, confirmar con una segunda prueba treponémica. ${ }^{23,31}$

La Organización Mundial de la Salud ha resaltado el uso de las pruebas rápidas, ampliamente utilizadas para la detección de $\mathrm{VIH}$ : no requieren un laboratorio, el entrenamiento y la interpretación son sencillos y se tienen resultados máximo en 30 minutos. ${ }^{32}$ En las normas oficiales mexicanas relativas al embarazo y las ITS no se considera su empleo para la detección de sífilis, sin embargo, en la relativa a males congénitos se mencionan las pruebas rápidas para sífilis, lo que ejemplifica la necesidad de homogeneizar las normas oficiales mexicanas relacionadas con la SC. Existen también las pruebas duales, tiras inmunocromatográficas que detectan simultáneamente dos agentes infecciosos, como VIH y sífilis; la utilidad, sensibilidad y especificidad de este tipo de pruebas aún se encuentra en estudio. ${ }^{33}$

Otro aspecto importante en la prevención de la transmisión congénita es el seguimiento. La NOM-039SSA2-2014 señala que las mujeres positivas a sífilis deben tener seguimiento con pruebas cuantitativas a los tres, seis y 12 meses, y los recién nacidos de 
madres con antecedentes de sífilis a los dos, cuatro, seis y 12 meses. Las pautas internacionales consideran verificar si la mujer estuvo en tratamiento previo contra sífilis, además de tomar en cuenta la búsqueda de los contactos de la mujer en función del estadio de la sífilis, para ofertarles la prueba de sífilis y el tratamiento. ${ }^{28}$ En las normas oficiales mexicanas se menciona que debe realizarse el seguimiento de los contactos, pero sin el énfasis necesario para coadyuvar a detener la transmisión. Un punto más del seguimiento es la comprobación de SC en los mortinatos de las mujeres que cursaban con sífilis durante el embarazo; es probable que no se estén notificando todos los casos de SC en numerosos países, debido a que se carece de definiciones de SC que incluyan los mortinatos sifilíticos, ${ }^{16}$ tal como sucede en México.

La comparación y contraste de documentos nacionales e internacionales pone de manifiesto la necesidad de actualizar las normas respecto el protocolo detallado y específico en atención primaria, el seguimiento de la mujer, sus contactos sexuales y sus productos (incluso los mortinatos), y las nuevas pruebas diagnósticas, las pruebas rápidas y el algoritmo reverso, para coadyuvar a la eliminación de la transmisión maternoinfantil de sífilis.

\section{Agradecimientos}

Parte del presente trabajo se desarrolló con el apoyo FOSISS-CONACyT-2015-262303.

\section{Bibliografía}

1. Peeling RW, Mabey D, Kamb ML, Chen XS, Radolf JD, Benzaken AS. Syphilis. Nat Rev Dis Primers. 2017;12;3:17073.

2. Berman SM. Maternal syphilis: pathophysiology and treatment. Maternal syphilis: pathophysiology and treatment. Bull World Health Organ. 2004;82:433-438

3. PAHO. Elimination of mother-to-child transmission of HIV and syphilis: update 2015. Washington. EE. UU.: PAHO; 2015.

4. Secretaría de Salud [sitio web]. Anuarios de morbilidad 1984-2017. Disponible en: https://www.gob.mx/salud/acciones-y-programas/anuarios-de-morbilidad-1984-2017

5. Conde-González CJ, Valdespino JL, Juárez Figueroa LA, Palma O, Olamendi-Portugal M, Olaiz-Fernández G, et al. Prevalencia de anticuerpos antitreponémicos y características sociodemográficas de la población mexicana adulta en el año 2000. Salud Publica Mex. 2007:49:S412-S420.

6. Yáñez-Álvarez I, Conde-González CJ, Uribe-Salas FJ, Olamendi-Portugal ML, García-Cisneros S, Sánchez-Alemán MA. Maternal/child seroprevalence of antibodies against Treponema pallidum at four general hospitals in the state of Morelos, Mexico. Arch Med Res. 2012;43:571-577.

7. Noyola DE, Malacara-Alfaro O, Lima-Rogel V, Torres-Montes A. Seroprevalencia de sífilis en mujeres embarazadas en San Luis Potosí. Salud Publica Mex. 2006;48:151-154.

8. Zamilpa-Mejía LG, Uribe-Salas F, Juárez-Figueroa L, Calderón-Jaimes E, Conde-González CJ. Prevalencia y factores asociados con sífilis y herpes genital en dos grupos de población femenina. Salud Publica Mex. 2003;45:S617-S623.
9. Juárez-Figueroa LA, Meléndez-Betancourt LA, Conde-González CJ. Hallazgo de sífilis a término del embarazo en mujeres de Cuernavaca, Mor. Rev Invest Clin. 2001;53:375-377.

10. Organización Panamericana de la Salud. Iniciativa regional para la eliminación de la transmisión maternoinfantil de VIH y de la sífilis congénita en América Latina y el Caribe. Documento conceptual. Uruguay: Centro Latinoamericano de Perinatología/Salud de la Mujer y Reproductiva/Organización Panamericana de la Salud/Organización Mundial de la Salud; 2009. D

11. Organización Panamericana de la Salud. ETMI-plus: marco para la eliminación de la transmisión maternoinfantil del VIH, la sífilis, la hepatitis y la enfermedad de Chagas; Uruguay: Organización Panamericana de la Salud/Organización Mundial de la Salud; 2017.

12. Organización Panamericana de la Salud. Eliminación de la transmisión maternoinfantil del VIH y la sífilis en las Américas. Actualización 2016. EE. UU.: Organización Panamericana de la Salud; 2017.

13. Norma Oficial Mexicana NOM-007-SSA2-2016, para la atención de la mujer durante el embarazo, parto y puerperio. México: Diario Oficial de la Federación; 2016.

14. Norma Oficial Mexicana NOM-039-SSA2-2014, para la prevención y control de las infecciones de transmisión sexual. México: Diario Oficial de la Federación; 2017.

15. Norma Oficial Mexicana NOM-010-SSA2-2018, para la prevención y el control de la infección por virus de la inmunodeficiencia humana. México: Diario Oficial de la Federación; 2018.

16. Norma Oficial Mexicana NOM-017-SSA2-2012, para la vigilancia epidemiológica. México: Diario Oficial de la Federación; 2013.

17. Norma Oficial Mexicana NOM-034-SSA2-2013, para la prevención y control de los defectos al nacimiento. México: Diario Oficial de la Federación; 2014.

18. Norma Oficial Mexicana NOM-039-SSA2-2014, para la prevención y control de las infecciones de transmisión sexual. México: Diario Oficial de la Federación; 2017

19. Norma Oficial Mexicana NOM-046-SSA2-2005. Violencia familiar, sexual y contra las mujeres. Criterios para la prevención y atención. México: Diario Oficial de la Federación; 2009.

20. Norma Oficial Mexicana NOM-253-SSA1-2012, para la disposición de sangre humana y sus componentes con fines terapéuticos. México: Diario Oficial de la Federación; 2012.

21. Guía de práctica clínica. Enfermedades de transmisión sexual en el adolescente y adulto que producen úlceras genitales: herpes, sífilis, chancroide, linfogranuloma venéreo y granuloma inguinal. México: Secretaría de Salud; 2009.

22. Diagnóstico, tratamiento y seguimiento de la sífilis congénita en el primero y segundo nivel de atención. México: Secretaría de Salud; 2011.

23. Workowski KA, Bolan G. Sexually Transmitted Diseases Treatment Guidelines, 2015. MMWR Recomm Rep. 2015;64:34-50.

24. Prevención, diagnóstico y tratamiento en el binomio madre-hijo con infección por el VIH. México: Secretaría de Salud; 2016.

25. Álvarez-Hernández G, Salazar-Arriola SA, Bocanegra-Luna C. Guía para el diagnóstico y manejo de la sífilis en el embarazo, y prevención de la sífilis congénita. México: Secretaría de Salud Pública del Estado de Sonora; 2012.

26. Pan American Health Organization. Syphilis testing practices in the Americas region: results of the 2014 survey. EE. UU.: Pan American Health Organization/World Health Organization; 2016.

27. Nielsen-Aage $H$, Reyn A. The Treponema pallidum immobilization test. Bull World Health Organ. 1956;14:263-263-288.

28. Gayet-Ageron A, Lautenschlager S, Nine B, Perneger TV, Combescure C. Sensitivity, specificity and likelihood ratios of PCR in the diagnosis of syphilis: a systematic review and meta-analysis. Sex Transm Infect. 2013;89:251-256.

29. Peeling RW, Ye H. Diagnostic tools for preventing and managing maternal and congenital syphilis: an overview. Bull World Health Organ. 2004;82:439-446.

30. National Center for HIV/AIDS, Viral Hepatitis, STD, and TB Prevention. Annual Report. EE. UU.: Centers for Disease Control and Prevention; 2016

31. Janier M, Hegyi V, Dupin N, Unemo M, Tiplica GS, Potočnik M, et al. 2014 European guideline on the management of syphilis. J Eur Acad Dermatol Venereol. 2014;28:1581-1593.

32. The Sexually Transmitted Diseases Diagnostics Initiative, Special Programme for Research \& Training in Tropical Diseases. The use of rapid syphilis tests. Suiza: United Nations Children's Fund/United Nations Development Programme/World Bank/World Health Organization; 2006.

33. Gliddon HD, Peeling RW, Kamb ML, Toskin I, Wi TE, Taylor MM. A systematic review and meta-analysis of studies evaluating the performance and operational characteristics of dual point-of-care tests for HIV and syphilis. Sex Transm Infect. 2017;93:S3-S15. 